\title{
Enhancement of performance of a hybrid SAC-OCDMA system using dynamic cyclic shift code
}

\author{
Abd T. H., Aljunid S. A., Fadhil H. A., Ahmad R. B. and Junita M. N. \\ School of Computer and Communication Engineering, \\ University Malaysia Perlis, Malaysia
}

Received: 09.10.2011

\begin{abstract}
A hybrid subcarrier multiplexing (SCM) technique incorporating spectral amplitude coding and optical code division multiple access (SAC-OCDMA) technologies is investigated using both numerical and simulation methods. The hybrid SCM/SAC-OCDMA system combines the two above schemes in such a way that it is robust to multiple access interference and reveals much enhanced channel data rate. We improve the overall performance of the hybrid SCM/SAC-OCDMA system based on spectral amplitude coding to suppress a number of impairments and improve its sensitivity at a bit error rate of $10^{-9}$. As a result, the system suggested by us, which is based on a dynamic cyclic shift code, shows better performance when compared with the standard SAC-OCDMA systems employing the conventional code.
\end{abstract}

Keywords: dynamic cyclic shift code, spectral amplitude coding - optical code division multiple access systems, phase induced intensity noise, modified quadratic congruence code, random diagonal code.

PACS: 42.79.Sz, 42.79.-e, 42.79.Ta

UDC: $535.317 .2 ; 535.318$

\section{Introduction}

Extremely large bandwidths and low propagation losses revealed by optical fibres are the two main reasons why optical local area networks (LAN) have revolutionised telecommunications. With the present level of technology, however, applications of the LAN are mainly limited by high costs of their optical components [1]. Optical code division multiple access (OCDMA) has always been of concern because of its inherent capability to support asynchronous-access networks, dynamic bandwidth assignment, and ability to support multimedia services $[2,3]$. Furthermore, the OCDMA has already been employed for the LAN and for different network access applications [4-6].

Nonetheless, the OCDMA systems suffer from different noises such as a shot noise, thermal noise, a dark current, and a phase-induced intensity noise (PIIN). In addition, multiple access interference (MAI) associated with availability of many users is considered as a dominating system-degradation factor for the OCDMA networks. Therefore, intelligent design of the code sequence is important when reducing contribution of the MAI to the total optical power received [7]. Accordingly, spectral amplitude coding and optical code division multiple access (SACOCDMA) technologies have been introduced, to result in good solutions which have reduced the effect of MAI by utilising codes with fixed in-phase cross-correlation [8]. Many different codes have been considered for the SAC-OCDMA networks. These include, in particular, an optical orthogonal code [9], a prime code [10], a Khazani-Syed (KS) code [11], an enhanced double 
weight (EDW) code [12], a modified frequency hopping (MFH) code [13], a modified quadratic congruence (MQC) code [14], a random diagonal (RD) code [15], and a modified double weight (MDW) code [16]. However, all of these codes suffer from some limitations. For instance, the code length in some cases is too long (especially for the OOC, KS, and EDW), the code construction is limited by the code parameter (e.g., for the MQC and MFH codes), and the crosscorrelation increases with increasing weight number (e.g., for the prime and RD codes).

Recently, a multi-diagonal (MD) code [17] has been suggested for the SAC-OCDMA systems of large cardinalities. It is designed such that the cross-correlation effect can be zero. However, construction of the MD code for a large number of users with zero cross-correlation would result in too long code lengths. The MD code families are being challenged by several problems such as rather restricted number of available code weights and time-consuming code construction. Code designs with a zero cross-correlation are required for the OCDMA systems since there is no more overlapping between the code sequences, which removes the effect of the MAI and suppresses the impact of the PIIN in such systems. Consequently, the effects of thermal and shot noises are only considered for the systems based on the zero cross-correlation code.

Here we suggest a dynamic cyclic shift (DCS) code. This code is closer to any real practical environment when compared to the MD code family. The DCS code is designed basing on the Galois field and the cyclic shift methods. The new code has a number of advantages such as (1) low cross-correlation $\left(\lambda_{c} \leq 1\right)$ that suppresses the MAI; (2) flexibility in choosing the weight parameter $W$ concerned with chips that correspond to a unit value and the $K$ parameter (a number of active users), thus giving superiority to the other codes like the MQC, KS and OOC ones; (3) simple design; (4) support of a large number of users; and (5) less code length comparing to the MD code. In addition, to study performance of the system with the DCS code, we will take into account the effect of different noises such as the PIIN, the shot and thermal noises).

A technology of subcarrier multiplexing (SCM) has already demonstrated its great potential to become a convenient and efficient technique for transmission of analogue or digital information [1]. Therefore hybrid SCM-OCDMA networks that employ the two efficient techniques, SCM and OCDMA, should be of a particular interest. The SCM systems have significant advantages since the microwave devices are more mature than the optical ones, while the frequency selectivity of microwave filters is much higher than that of their optical counterparts. In addition, owing to lowcost system implementation typical of the SCM, the latter has been proposed to transmit multichannel digital optical signals in the LAN [18].

In this article we suggest a new technique for improving the performance of the SCM/SACOCDMA system. Our system is evaluated basing on the DCS code, in order to mitigate the MAI effects and increase the number of optical channels. An AND detection technique discussed in details in the study [12] is used for detecting optical signals, thus reducing the cost and complexity and improving the overall performance of the system. We investigate the performance of our hybrid system numerically and with an optical simulator software "OptiSim ${ }^{T M}$ ", taking into consideration the effects of inter-modulation distortion noise. The remainder of this article is organised as follows. In Section 2 we focus upon the construction of the DCS code. Section 3 presents configuration of a hybrid SCM/SAC-OCDMA system and Section 4 analyses performance of that system. Section 5 is devoted to simulation model of our system and analysis of its performance. Finally, conclusions are drawn in Section 5.

Ukr. J. Phys. Opt. 2012, V13, №1 


\section{Construction of dynamic cyclic shift code}

We have developed a new code referred to as DCS, which includes the parameters $N, W$ and $\lambda_{c}$, where $N$ denotes the code length (i.e., the number of total chips), $W$ the code weight, and $\lambda_{c}$ indicates the in-phase cross-correlation.

The cross-correlation $\lambda_{c}$ between any pair of code sequences must be small enough. This property would ensure that each code sequence can be easily distinguished from every other address sequence. In other words, we seek to make the MAI which remains insignificant when compared to the energy contained in the information received. For code sequences $X=\left(x_{1}, x_{2}, \ldots x_{N}\right)$ and $Y=\left(y_{1}, y_{2}, \ldots \ldots . y_{N}\right)$, the cross-correlation is given by $\lambda_{c}=\sum_{i=1}^{N} x_{i} y_{i}$.

The codes with ideal in-phase cross-correlation $\left(\lambda_{c} \leq 1\right)$ are required in the OCDMA systems since these codes eliminate multi-user interference and suppress the effect of the PIIN. The technique utilised for constructing the DCS code is detailed further below.

\subsection{Algorithm for DCS code design}

The new code family suggested here is represented as $\left(N=\sum_{i=1}^{W-1} 2^{i}+D, W, \lambda_{c}\right)$, where $i=\{0,1, \ldots, W-1\}$, denotes a positive integer number and $D$ represents the dynamic part. The next steps are followed to construct the DCS code words:

$\underline{\text { Step } 1}$

First we construct a sequence $S^{i}$ of integer numbers that are elements of the Galois field $\operatorname{GF}(N)=\{1,2, \ldots ., N\}$ over an integer number $N$, using the expression

$$
S^{i}=\left\{\begin{array}{l}
\left(2^{i}\right)(\bmod N), i=0,1 \\
\left(S^{i-1}+2^{i}\right)(\bmod N), i=2,3, \ldots ., W-1 .
\end{array}\right.
$$

Here $S^{i}, N$, and $W$ are the elements over the Galois field $G F(N)$.

$\underline{\text { Step } 2}$

After that we construct a sequence $T_{i}$ of binary numbers $(0,1)$ basing on the generated sequence $S^{i}$ and using the mapping method,

$$
T_{i}=\left\{\begin{array}{ll}
1, & \text { for } S^{i} \\
0, & \text { otherwise }
\end{array} .\right.
$$

$\underline{\text { Step } 3}$

Now we combine the binary sequence of each $T_{i}$ that has been generated in the step 2, to get the first code sequence of the first user. The elements of the first code word are given by

$$
C_{1}=T_{0}+T_{1}+T_{2}+\ldots \ldots+T_{W-1} .
$$

The method for producing the binary sequence $(0,1)$ based on $G F(N)$ is shown in Fig. 1. We have also illustrated generation of the first DCS code word sequence in Table 1 for the code parameters $W=3, D=8$ and $N=22$ as an example. In Table $1, C_{1}$ denotes the first code word of the DCS code. In Fig. 2 we display a procedure of combining the sequences $T_{i}$ needed for generating the first code word of the DCS code. 
Table 1 . Generation of sequence $S^{i}$ and binary sequence $T_{i}$ for the case of $W=4, D=8$ and $N=22$.

\begin{tabular}{|c|c|c|}
\hline$i$ & $S^{i}$ & $T_{i}$ \\
\hline 0 & 1 & 1000000000000000000000 \\
\hline 1 & 2 & 0100000000000000000000 \\
\hline 2 & 6 & 0000010000000000000000 \\
\hline 3 & 14 & 0000000000000100000000 \\
\hline \multicolumn{2}{|c|}{$C_{1}$} & 1100010000000100000000 \\
\hline
\end{tabular}

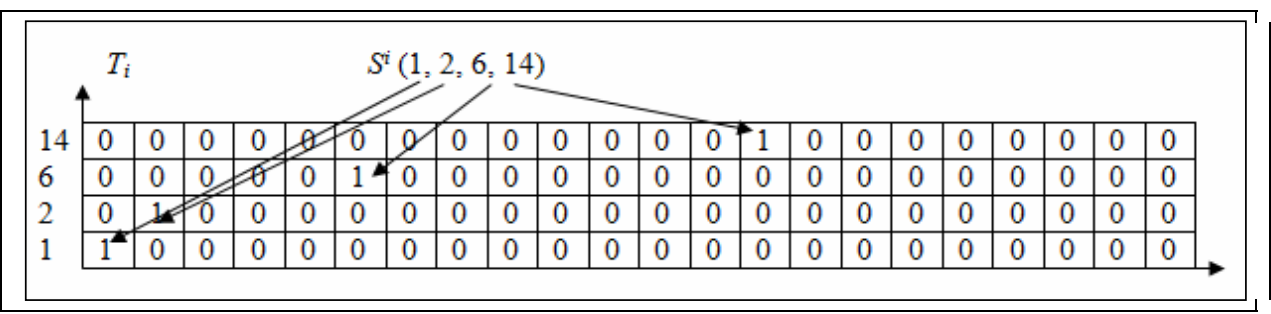

Fig. 1. Generation of sequence $T_{i}$ for the case of $N=22, D=8$ and $W=4$.

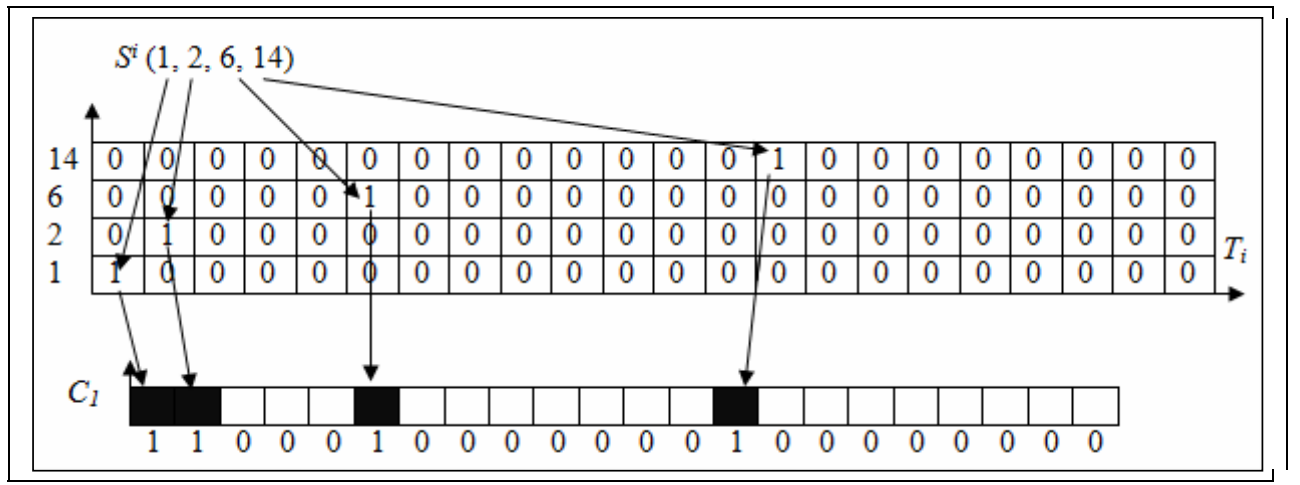

Fig. 2. Combination of sequences $T_{i}$ used for producing the first code word.

After generating the first code sequence, we use a cyclic shift method to produce the code sequence for the rest of the users. There are $N-1$ different sequences which can be generated from the first code sequence. The number of the code sequences is equal to the code length $(K=N)$. In order to achieve the cyclic shift property with a minimum cross-correlation $\left(\lambda_{c} \leq 1\right)$, we always consider the $D$ values given by the inequality $D>7$. Otherwise, the cross-correlation value would have been larger than one.

In our code, the number of users can be increased by increasing the dynamic sequence $D$ only, while the weight sequence part remains unchanged. An example is presented in Table 2 for $K=14$ subscribers, where $W=3$ and $D=8$.

Table 3 shows the weight $W$, the code length $N$, and the cross-correlation $\lambda_{c}$ for 30 users. It is clearly seen that the DCS code can be generated with lower code weights $(W=2)$ and less lengths, when compared to the other codes. It is also seen that the DCS code reveals better crosscorrelation values $\left(\lambda_{c} \leq 1\right)$. Furthermore, if the chip width (i.e., the filter bandwidth) of $0.8 \mathrm{~nm}$ is used, the spectral widths required for the OOC, RD, MDW, MQC, KS, MFH and DCS codes are 291.2, 28, 44.8, 72, 115.2, 33.6 and $24 \mathrm{~nm}$, respectively. As evident from Table 3, there is no other code that can be generated with the code weight equal to two, except of the DCS code.

Ukr. J. Phys. Opt. 2012, V13, №1 
Table 2. DCS code words for the case of $W=3, D=8$ and $K=14$.

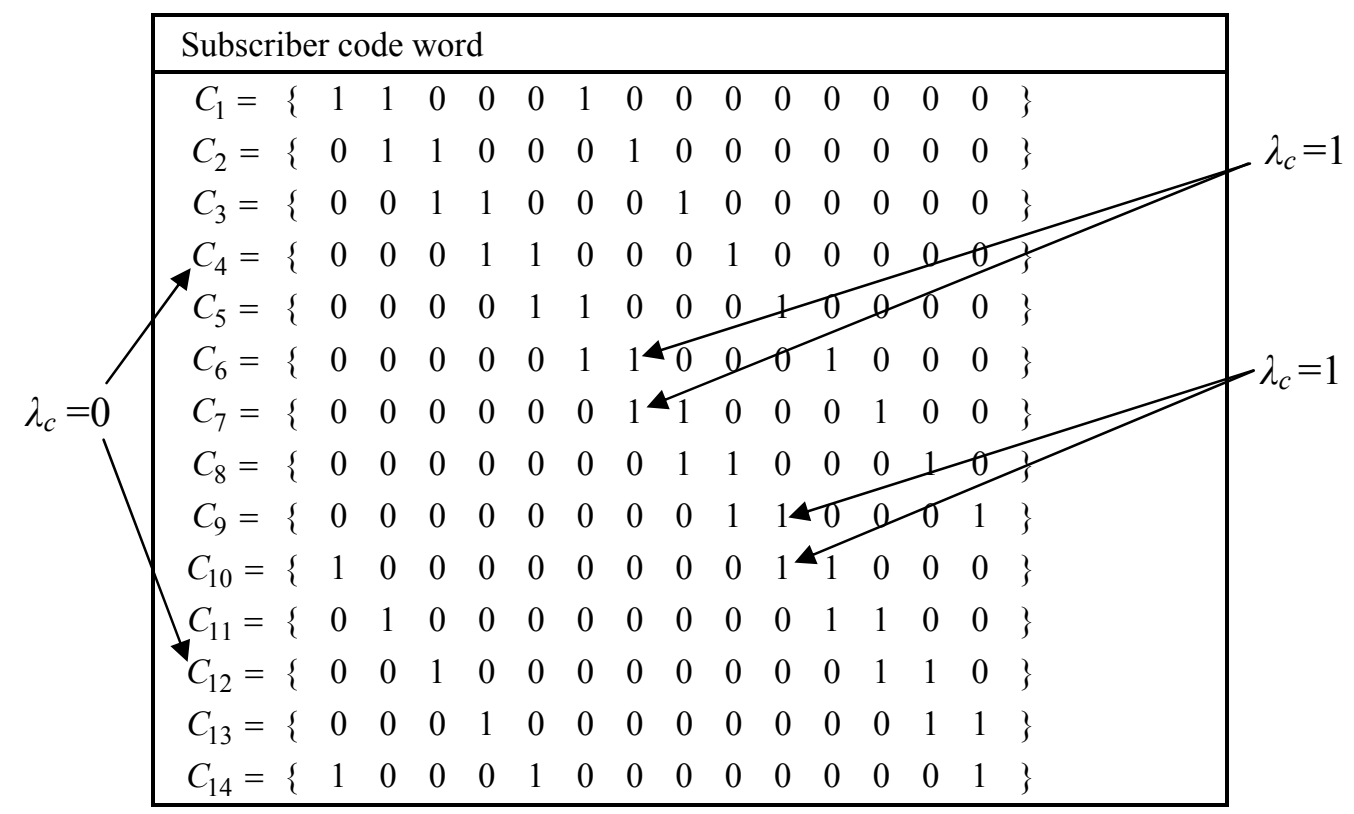

Table 3. Comparison of different codes used for the SAC-OCDMA system.

\begin{tabular}{cccccc}
\hline Number & Codes & $\begin{array}{c}\text { Number of } \\
\text { users } K\end{array}$ & Weight $W$ & Code length $N$ & $\begin{array}{c}\text { Cross-correlation } \\
\lambda_{c}\end{array}$ \\
\hline 1 & OOC & 30 & 4 & 364 & 1 \\
2 & RD & 30 & 4 & 35 & $\begin{array}{c}\text { Variable } \lambda_{c} \text { in the } \\
\text { code segment }\end{array}$ \\
3 & MQC & 30 & 8 & 56 & 1 \\
4 & MDW & 30 & 4 & 90 & 1 \\
5 & KS & 30 & 4 & 144 & 1 \\
6 & MFH & 30 & 7 & 42 & $\leq 1$ \\
7 & DCS & 30 & 2 & 30 &
\end{tabular}

\section{Hybrid SCM/SAC-OCDMA system configuration}

In this section we demonstrate a hybrid SCM/SAC-OCDMA based on the DCS code. Fig. 3 illustrates schematic diagram of the SCM/SAC-OCDMA transceiver system. In a transmitter part, electrical data are modulated with subcarrier frequencies. All the modulated subcarriers are then combined using an electrical adder. The optical external modulator is then used to modulate the combined modulated subcarrier frequencies with a distinct code sequence. On the other hand, each code word of the SAC-OCDMA based on the DCS code consists of different wavelengths. An optical multiplexer has been used for combining all the modulated code sequences and sending them out through single-mode optical fibre.

In a receiver part, a de-multiplexer is used to separate different modulated code words. A code sequence received is filtered out with a fibre Bragg grating filter based on the AND subtraction technique. The signals are thus de-multiplexed, decoded and separated by the AND detection technique. After a photo-detector, electrical splitter and bandpass filter are used to split the SCM signals and reject the unwanted signals. To retrieve the data sent originally, a local 
microwave frequency $f_{i}$ is mixed electrically with the incoming signal and then filtered by a lowpass filter.

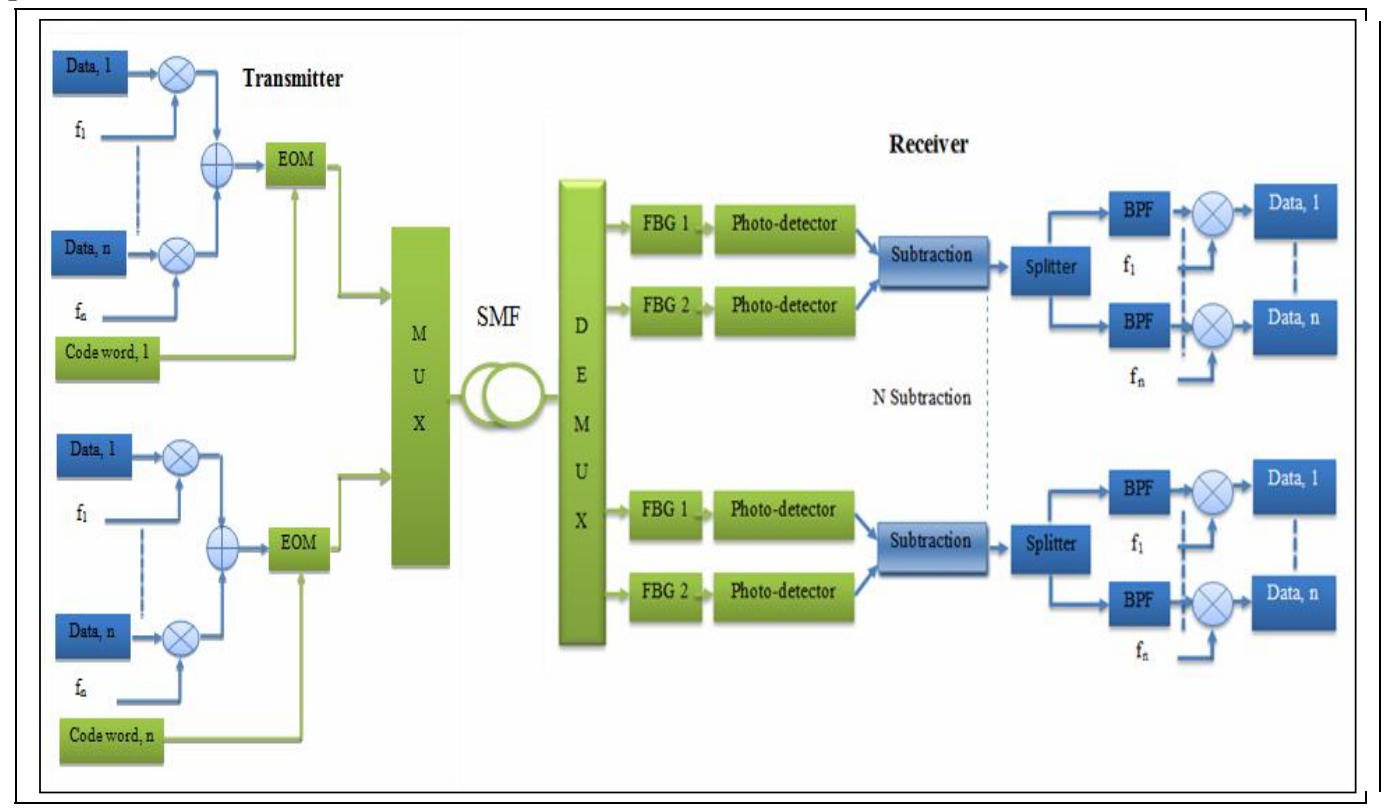

Fig. 3. A block diagram of SCM/SAC-OCDMA system that uses DCS code and AND detection technique.

\section{System performance analysis}

\subsection{AND subtraction technique}

The AND subtraction technique [12] has been used as a detection method. This technique is fully capable of eliminating the MAI, reducing complexity of receiver and improving performance of the system. In the frame of this technique, a spectral amplitude signal at the receiver side is split into two branches. The upper branch is the signal for a user $\mathrm{X}$ associated with cross-correlation between $\mathrm{X}$ and $\mathrm{Y}$, and the lower branch should be a cross-correlation result from the AND operation between $\mathrm{X}$ and $\mathrm{Y}$, which have the same cross-correlation magnitude associated with the signal in the upper branch. The technique can be best described using Fig. 4.

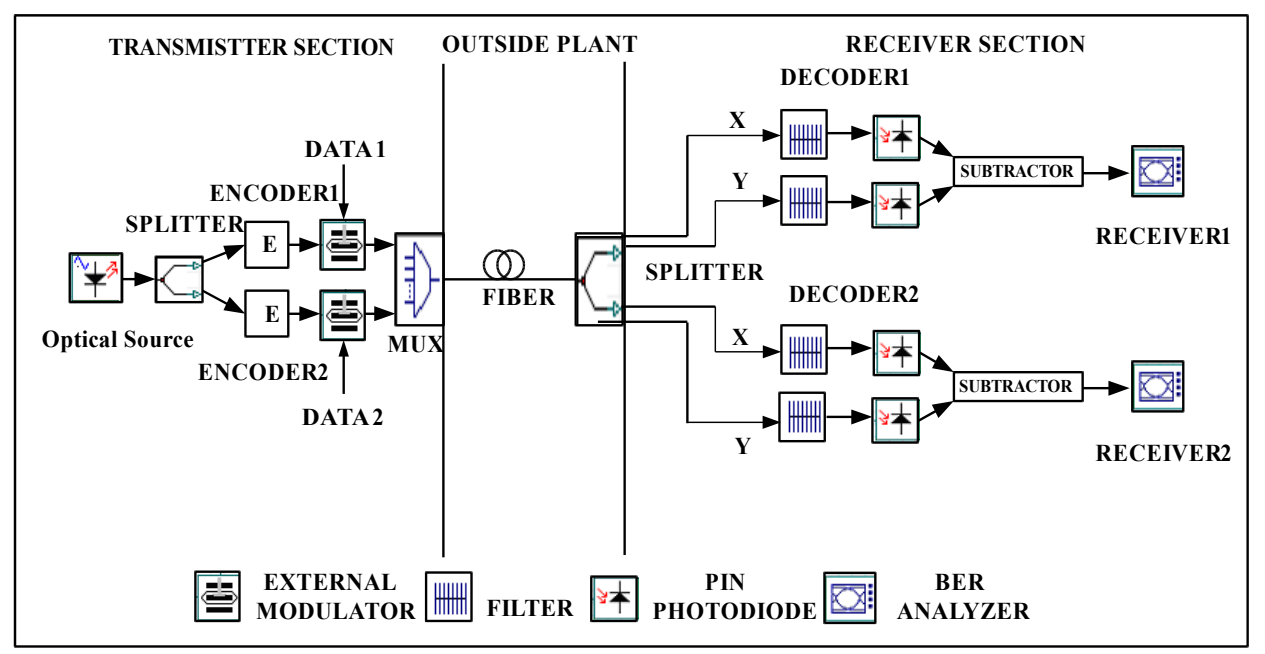

Fig. 4. Schematic diagram of AND detection scheme used for the DCS code. 


\subsection{Bit error rate performance analysis}

We consider the effects of an incoherent intensity noise $\left\langle i_{P I I N}^{2}\right\rangle$, a shot noise $\left\langle i_{\text {shot }}^{2}\right\rangle$, an intermodulation distortion $\left\langle i_{I M D}^{2}\right\rangle$ in a photo-detector, and a thermal noise $\left\langle i_{\text {thermal }}^{2}\right\rangle$. The detection scheme employed for our system is based on the AND detection technique that uses a fibre Bragg grating followed by a photo-detector. A Gaussian approximation can be used when calculating the bit error rate (BER).

When incoherent light fields are mixed and incident upon a photo-detector, the phase noise of those fields causes an intensity noise in the photo-detector output [13]. Here the source coherence time $\tau_{c}$ is expressed as [15]

$$
\tau_{c}=\frac{\int_{0}^{\infty} G^{2}(v) d v}{\left[\int_{0}^{\infty} G(v) d v\right]^{2}}
$$

where $G(v)$ denotes the single-sideband power spectral density of the thermal source. The Q-factor performance provides qualitative description of performance of the optical receiver. The latter should depend on the signal-to-noise ratio (SNR). The Q-factor suggests a minimum SNR required to obtain a specific BER for a given signal [15]. The SNR of an electrical signal is defined as an average signal-to-noise power $S N R=\left[I^{2} / \sigma^{2}\right]$, where $\sigma^{2}$ is the variance of the noise source (notice that the effects of dark current of the receiver and amplification noises are neglected in our analysis), which is given by

$$
\sigma^{2}=\left\langle i_{\text {shot }}^{2}\right\rangle+\left\langle i_{\text {PIIN }}^{2}\right\rangle+\left\langle i_{\text {thermal }}^{2}\right\rangle+\left\langle i_{\text {IMD }}^{2}\right\rangle .
$$

The first, second and the third terms in the r. h. s. of Eq. (7) result from the shot noise, the effect of the PIIN and the thermal noise, respectively. The fourth term represents the intermodulation distortion in the subcarrier channels in the both photo-detectors. Let $C_{K}(i)$ denote the $i$ th element of the $K$ th DCS code sequence. The code properties for the AND subtraction technique can therefore be written as

$$
\sum_{i=1}^{N} C_{K}(i) C_{l}(i)= \begin{cases}W, & \text { for } K=l, \\ 1, & \text { for } K \neq l \text { and } C_{K}(i) C_{l}(i)=1, \\ 0, & \text { for } K \neq l \text { and } C_{K}(i) C_{l}(i)=0\end{cases}
$$

and

$$
\sum_{i=1}^{N} C_{K T}(i)\left(C_{K}(i) \bullet C_{l}(i)\right)=\left\{\begin{array}{l}
1, \text { for } K=l, \text { and } K T=\left\{\begin{array}{l}
K+1, \text { if } K<l, \\
K-1, \text { if } K>l,
\end{array}\right. \text { The AND operation of } \\
1, \text { for } K \neq l, \text { and } K T=\left\{\begin{array}{l}
K+1, \text { if } K<l, \\
K-1, \text { if } K>l .
\end{array}\right.
\end{array}\right.
$$

$C_{K T}(i)\left(C_{K}(i) \bullet C_{l}(i)\right)$ is valid for $K=l$ and $K \neq l$ (see Eq. (8)), while the cross-correlation of $C_{K}(i) C_{l}(i)$ is $W$ if $K=l$. The MAI can be fully eliminated when subtracted from $C_{K T}(i)\left(C_{K}(i) \bullet C_{l}(i)\right)$ for the case of $K \neq l$. Then we obtain

$$
\sum_{i=1}^{N} C_{K}(i) C_{l}(i)-\sum_{i=1}^{N} C_{K T}(i)\left(C_{K}(i) \bullet C_{l}(i)\right)=\left\{\begin{array}{l}
W-1, \text { for } K=l \\
0, \text { for } K \neq l .
\end{array}\right.
$$


Hence, the weight is zero when $K \neq l$, thus meaning that the MAI is fully eliminated if the AND detection technique is used.

To analyse the system that includes both transmitter and receiver, we assume that the spacing of optical channels is significantly wide so that the effect of cross-talk from the adjacent optical channels is negligible [19]. Furthermore, the subcarrier channels have the same speed. In addition to the same assumption as used in $[13,15]$, in the analysis of our system we suppose the following:

1. Each light source is ideally unpolarised and its spectrum is flat outside the bandwidth $\left[v_{0}-\Delta v / 2, v_{0}+\Delta v / 2\right]$ where $v_{0}$ is the central optical frequency and $\Delta v$ the bandwidth of optical source expressed in Hertz.

2. Each power spectral component has an identical spectral width.

3. Each user gets the same power at the receiver.

4. Bit streams from each user are synchronised.

The above assumptions are important for mathematical simplification of the system, which can easily be analysed using the Gaussian approximation. Otherwise, it would have been difficult to evaluate the system performance.

The power spectral density of the received optical signals can be written as [13, 14]

$$
r(v)=\frac{P_{s r}}{\Delta v} \sum_{K=1}^{K} d_{K} \sum_{i=1}^{N} c_{K}(i) \operatorname{rect}(i),
$$

where $P_{s r}$ is the effective power of a broadband source at the receiver, $K$ the number of active users, $N$ the DCS code length, $d_{K}$ the data bit of $K$ th user which is " 1 " or " 0 ", and $d_{K}$ denotes the modulation data of $n$th subcarrier channel in the Kth optical code word, which may be expressed as [19]

$$
d_{k}(t)=\sum_{n=1}^{N c} u_{n, K}(t) m_{n, K} \cos \left(w_{n} t\right) .
$$

Here $u_{n, K}(t)$ is the normalised digital signal in the $n$th subcarrier channel of the $K$ th code word, $w_{n}$ the angular subcarrier frequency, $m_{n, K}$ the modulation index of the $n$th subcarrier of $K$ th user, and $N_{c}$ the number of subcarrier channels in each code word [19-21]. Let us assume an identical modulation index for all the subcarrier channels:

$$
0 \leq m_{n, K} \leq \frac{1}{N_{S}} .
$$

The $\operatorname{rect}(i)$ function in Eq. (11) is given by

$$
\operatorname{rect}(i)=u\left[v-v_{0}-\frac{\Delta v}{2 N}(-N+2 i-2)\right]-u\left[v-v_{0}-\frac{\Delta v}{2 N}(-N+2 i)\right]=u\left[\frac{\Delta v}{N}\right],
$$

where $u(v)$ is the unit step function:

$$
u(v)= \begin{cases}1, & v \geq 0 \\ 0, & v<0\end{cases}
$$

The total incident powers at the input of some positive intrinsic negatives PIN 1 and PIN 2 (see Fig. 4) are given by

$$
\int_{0}^{\infty} G_{1}(v) d v=\int_{0}^{\infty}\left[\frac{P_{s r}}{\Delta v} \sum_{K=1}^{K} d_{K} \sum_{i=1}^{N} C_{K}(i) C_{l}(i)\left\{u\left[\frac{\Delta v}{N}\right]\right\}\right] d v=\frac{P_{s r} W}{N}+\frac{P_{s r}}{N} \sum_{K=1, K \neq l}^{K} d_{K}
$$


and

$$
\begin{aligned}
\int_{0}^{\infty} G_{2}(v) d v & =\int_{0}^{\infty}\left[\frac{P_{s r}}{\Delta v} \sum_{K=1}^{K} d_{K} \sum_{i=1}^{N} C_{K T}(i)\left(C_{K}(i) \bullet C_{l}(i)\right)\left\{u\left[\frac{\Delta v}{N}\right]\right\}\right] d v \\
& =\frac{P_{s r}}{N}+\frac{P_{s r}}{N} \sum_{K=1, K \neq l}^{K} d_{K} .
\end{aligned}
$$

Since the signal from a desired user is obtained as a difference $I$ of the two photodiode currents, one can write

$$
I=I_{1}-I_{2},
$$

where $I_{1}, I_{2}$ denote the currents at the PIN 1 and the PIN 2, respectively. Combining Eqs. (15) and Eq. (16), we obtain

$$
I=\mathfrak{R}\left[\int_{0}^{\infty} G_{1}(v) d v-\int_{0}^{\infty} G_{2}(v) d v\right]=\frac{\mathfrak{R} P_{s r}(W-1)}{N} \sum_{n=1}^{N c} u_{n, K}(t) m_{n, K} \cos \left(w_{n} t\right),
$$

where $\mathfrak{R}$ means the response of the photo-detectors $\left(\mathfrak{R}=\eta e / h v_{c}\right), \eta$ the quantum efficiency, $e$ the electron charge, $h$ the Planck constant, and $v_{c}$ the central frequency of the original broadband optical pulse. At the RF demodulator, the signal is coherently mixed with a local oscillator $2 \cos \left(w_{n} t\right)$ [19]. As a result, Eq. (18) reduces to

$$
\begin{aligned}
& I=\frac{\mathfrak{R} P_{S r}(W-1)}{N} \sum_{n=1}^{N c} u_{n, K}(t) m_{n, K} \cos \left(w_{n} t\right)\left[2 \cos \left(w_{n} t\right)\right], \\
& I=\frac{\mathfrak{R} P_{S r}(W-1)}{N} \sum_{n=1}^{N c} u_{n, K}(t) m_{n, K}\left[1+\cos \left(2 w_{n} t\right)\right] .
\end{aligned}
$$

Since the double-frequency component is filtered out with the low-pass filter, the modulator output will become

$$
I=\frac{\Re P_{s r}(W-1)}{N} u_{n, K}(t) m_{n, K} .
$$

The power of the shot noise can be expressed as

$$
\left\langle i_{\text {shot }}^{2}\right\rangle=2 e B\left(I_{1}+I_{2}\right)=2 e B \Re\left[\int_{0}^{\infty} G_{1}(v) d v+\int_{0}^{\infty} G_{2}(v) d v\right]=2 e B \Re\left[\frac{P_{s r}(W+3)}{N}\right] .
$$

Using the methodology similar to that used in Ref. [12] and approximating the summations as $\sum_{K=1}^{K} c_{K} \approx \frac{K W}{N}$ and $\sum_{K=1}^{K} c_{K T} \approx \frac{K W}{N}$, one can express the noise power as

$$
\begin{aligned}
\left\langle i_{P I I N}^{2}\right\rangle & =B I_{1}^{2} \tau_{C 1}+B I_{2}^{2} \tau_{C 2}, \\
\left\langle i_{P I I N}^{2}\right\rangle & =B \Re^{2}\left[\int_{0}^{\infty} G_{1}^{2}(v) d v+\int_{0}^{\infty} G_{2}^{2}(v) d v\right], \\
\left\langle i_{P I I N}^{2}\right\rangle & =B \Re^{2} \frac{P_{s r}^{2}}{N \Delta v} \sum_{i=1}^{N}\left\{C_{l}(i) \times\left[\sum_{K=1}^{K} d_{K} C_{K}(i)\right] \times\left[\sum_{m=1}^{K} d_{m} C_{m}(i)\right]\right\} \\
& +B \mathfrak{R}^{2} \frac{P_{s r}^{2}}{N \Delta v} \sum_{i=1}^{N}\left\{C_{K}(i) \bullet C_{l}(i) \times\left[\sum_{K=1}^{K} d_{K} C_{K T}(i)\right] \times\left[\sum_{m=1}^{K} d_{m} C_{m}(i)\right]\right\},
\end{aligned}
$$




$$
\begin{aligned}
&\left\langle i_{P I I N}^{2}\right\rangle \cong \frac{B \Re^{2} P_{s r}^{2}}{N \Delta v} \sum_{i=1}^{N}\left\{C_{l}(i) \times \frac{K W}{N} \times\left[\sum_{K=1}^{K} C_{K}(i)\right]\right\} \\
&+\frac{B \Re^{2} P_{s r}^{2}}{N \Delta v} \sum_{i=1}^{N}\left\{C_{K}(i) \bullet C_{l}(i) \times \frac{K W}{N} \times\left[\sum_{K=1}^{K} C_{K T}(i)\right]\right\} . \\
&\left\langle i_{P I I N}^{2}\right\rangle \cong \frac{B \Re^{2} P_{s r}^{2}}{N \Delta v} \times\left[\frac{K W}{N}\right] \sum_{K=1}^{K}\left[\sum_{i=1}^{N} C_{K}(i) C_{l}(i)\right] \\
&+\frac{B \Re^{2} P_{s r}^{2}}{N \Delta v} \times\left[\frac{K W}{N}\right] \sum_{K=1}^{K}\left[\sum_{i=1}^{N} C_{K T}(i)\left(C_{K}(i) \bullet C_{l}(i)\right)\right], \\
&\left\langle i_{P I I N}^{2}\right\rangle=\frac{B \Re^{2} P_{s r}^{2} K W(W+3)}{N^{2} \Delta v}
\end{aligned}
$$

The thermal noise is given as [13]

$$
\left\langle i_{\text {thermal }}^{2}\right\rangle=\frac{4 K_{b} T_{n} B}{R_{L}}
$$

where $e$ is the electron charge and $K_{b}$ the Boltzmann constant and the inter-modulation distortion is expressed as $[20,21]$

$$
I_{I M D}^{2}=P_{s r}^{2} \mathfrak{R}^{2} m_{n, K}^{6}\left[\frac{D_{1,1,1}}{32}+\frac{D_{2,1}}{64}\right] .
$$

Here $D_{1,1,1}$ is the three-tone third-order inter-modulation at $f_{i}+f_{K}-f_{l}$,

$$
D_{1,1,1}=\frac{N_{c}}{2}\left\{\left(N_{s}-N_{c}+1\right)+\frac{1}{4}\left\{\left(N_{s}-3\right)^{2}-5\right\}-\frac{1}{8}\left[1-(-1)^{N_{s}}\right](-1)^{N_{c}+N_{s}}\right\},
$$

and $D_{2,1}$ represents the two-tone third-order inter-modulation at $2 f_{i}-f_{K}$,

$$
D_{1,2}=\frac{1}{2}\left\{N_{s}-2-\frac{1}{2}\left[1-(-1)^{N_{s}}\right](-1)^{N_{C}}\right\} .
$$

From Eqs. (20)-(23) one can compute the average SNR of the SCM/SAC-OCDMA system for the DCS code, noticing that the probability of sending a bit ' 1 ' at any time for each user is $1 / 2$ [15]. Finally, the SNR of the AND detection system can be expressed as

$$
S N R=\frac{I^{2}}{\sigma^{2}}=\frac{\left(\frac{\Re P_{s r}(W-1)}{N}\right)^{2}}{\frac{e B \Re P_{s r}(W+3)}{N}+\frac{B \Re^{2} P_{s r}^{2} K W(W+3)}{N^{2} \Delta v}+P_{s r}^{2} \Re^{2} m_{n, K}^{6}\left[\frac{D_{1,1,1}}{32}+\frac{D_{2,1}}{64}\right]+\frac{4 K_{b} T_{n} B}{R_{L}}}
$$

Eq. (24) is a general relation used for calculating the SNR for the AND detection system and the DCS code. Since no pulses are sent for the data bit ' 0 ', the formula used to calculate the BER in the frame of Gaussian approximation stands as $[13,14]$

$$
B E R=\frac{1}{2} \operatorname{erfc}\left(\sqrt{\frac{S N R}{8}}\right) .
$$




\section{Numerical and simulation analyses}

\subsection{Numerical analysis}

The performance of the DCS code has been compared numerically with the recently suggested codes such as the KS code, the EDW code, the MFH code, and the MQC one. We evaluate the BER and the SNR using Eqs. (24) and (25), respectively. The parameters used in our numerical calculations are gathered in Table 4.

Table 4. Typical parameters used in our numerical analysis and calculations.

\begin{tabular}{|l|l|l|}
\hline Symbol & \multicolumn{1}{|c|}{ Parameter } & Value \\
\hline$\eta$ & Quantum efficiency of photo-detector & 0.6 \\
\hline$P_{s r}$ & Broadband effective power & $-10 \mathrm{dBm}$ \\
\hline$B$ & Electrical bandwidth & $80 \mathrm{MHz}$ \\
\hline$\lambda_{0}$ & Operating wavelength & $1550 \mathrm{~nm}$ \\
\hline$R_{b}$ & Data bit rate & $155 \mathrm{M} \mathrm{bit} / \mathrm{s}$ \\
\hline$T_{n}$ & Receiver noise temperature & $300 \mathrm{~K}$ \\
\hline$R_{L}$ & Receiver load resistor & $1030 \Omega$ \\
\hline
\end{tabular}

Fig. 5 shows the relationship between the number of simultaneous users and the BER for the DCS, KS, MFH, and MQC codes. It is clearly seen that the performance of the DCS code is much higher when compared to the others, even though the weight of the other codes is greater than that of the DCS one. The maximum acceptable BER is $10^{-9}$ that we achieved by the DCS code compared. We can ascertain from this fact that the DCS code has a small length, the number of the active users is equal to the code size and the cross-correlation is $\lambda_{c}=1$ or $\lambda_{c}=0$, while the other codes like MQC, MFH and KS have longer lengths. Specific parameter values have been chosen basing upon the results published for these practical codes [14, 19]. The BER calculated for the DCS code has been $W=4$ for the KS, $W=12$ for the MFH, and $W=14$ for the MQC code. However, the system that uses the DCS code can support 160 active users, whereas the KS, MQC and MFH can support 114, 91 and 58 users, respectively.

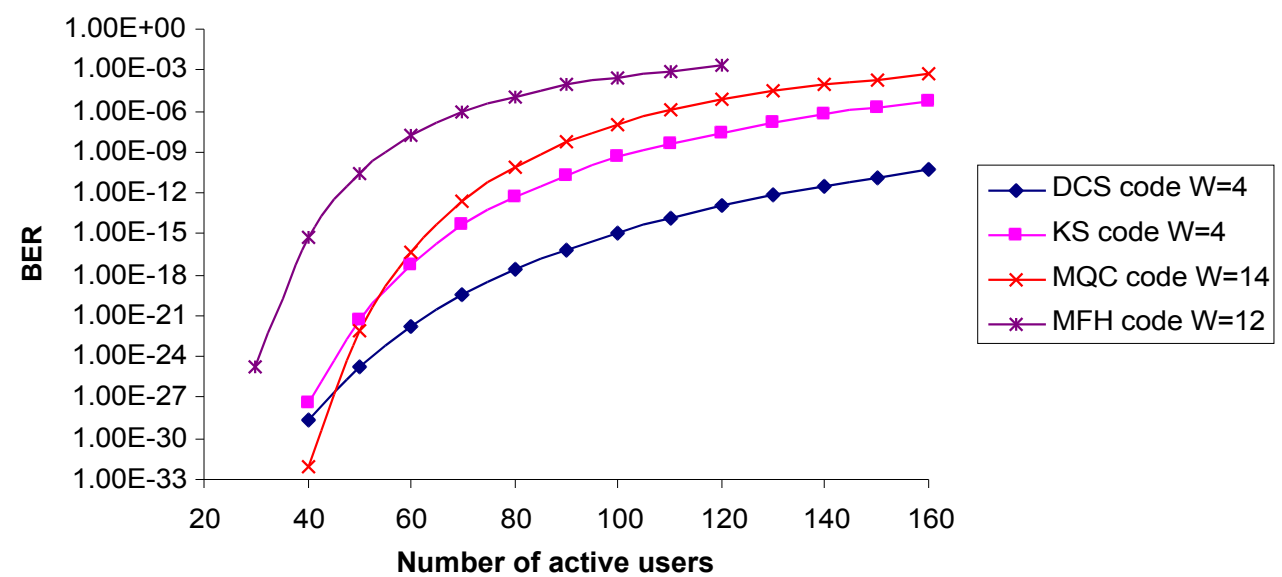

Fig 5. BER versus the number of active users for different codes employed in the frame of SAC-OCDMA technique $(155 \mathrm{Mb} / \mathrm{s})$. 
The dependences of the BER on the number of users are shown in Fig. 6 for the cases of the both DCS and KS codes employed by the SCM/SAC-OCDMA technique. These codes use the same AND detection technique to detect the optical signal. Fig. 6 testifies that the performance of the SCM/SAC-OCDMA system based on the DCS code is higher than that based on the KS code. Nonetheless, in order to make our comparison fairer, we compare the DCS-code system with the KS-code one under the conditions of the same code weight number. We find that the DCS-code system is better. Moreover, the performance of the DCS code is still higher, though the number of subcarriers is greater than that used in the hybrid KS-code system. This is achieved due to specific properties of the DCS code. Hence, the DCS code can be used when improving performance of the hybrid SCM/SAC-OCDMA systems.

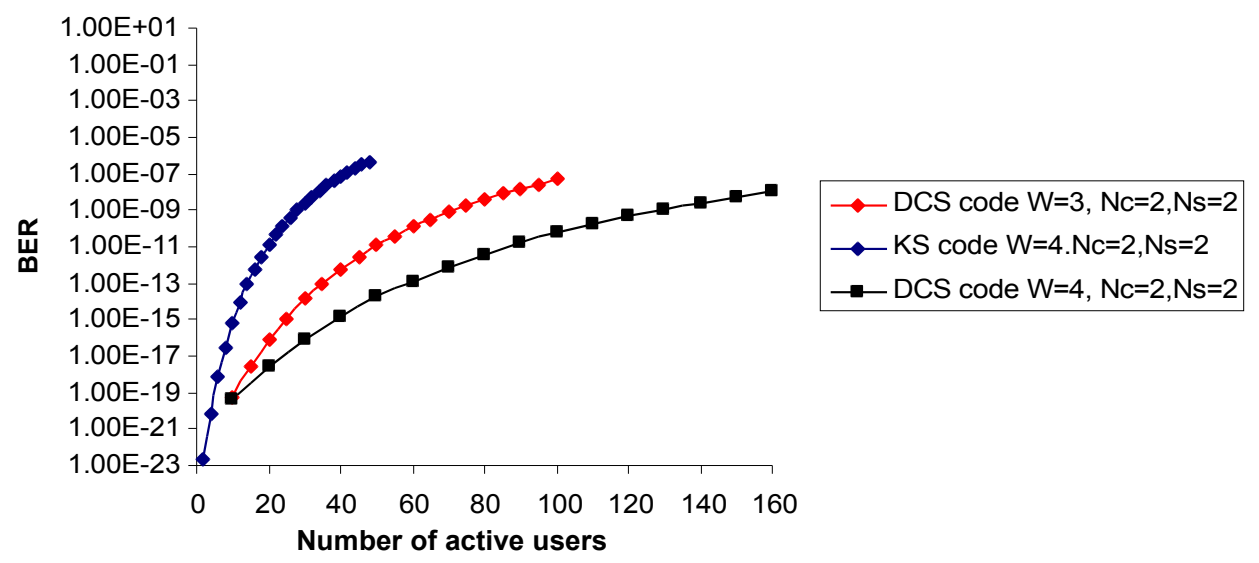

Fig. 6. BER versus the number of active users for the hybrid systems employing the DCS and $\mathrm{KS}$ codes $\left(P_{s r}=-10 \mathrm{dBm}\right)$.

Fig. 7 depicts the performance of the hybrid system that uses the DCS code for different data rates. The data are compared for the cases of $622 \mathrm{Mb} / \mathrm{s}$ and $1.25 \mathrm{~Gb} / \mathrm{s}$. In addition, the effective source power $\left(P_{s r}\right)$ is fixed here at the value $-10 \mathrm{dBm}$ in order to show the performance of the system at low effective-source power. Furthermore, the system is also tested for different weight numbers ( $W=5$ and $W=6$ ) of the DCS code, in order to observe the effect of the latter on the capability of the hybrid system.

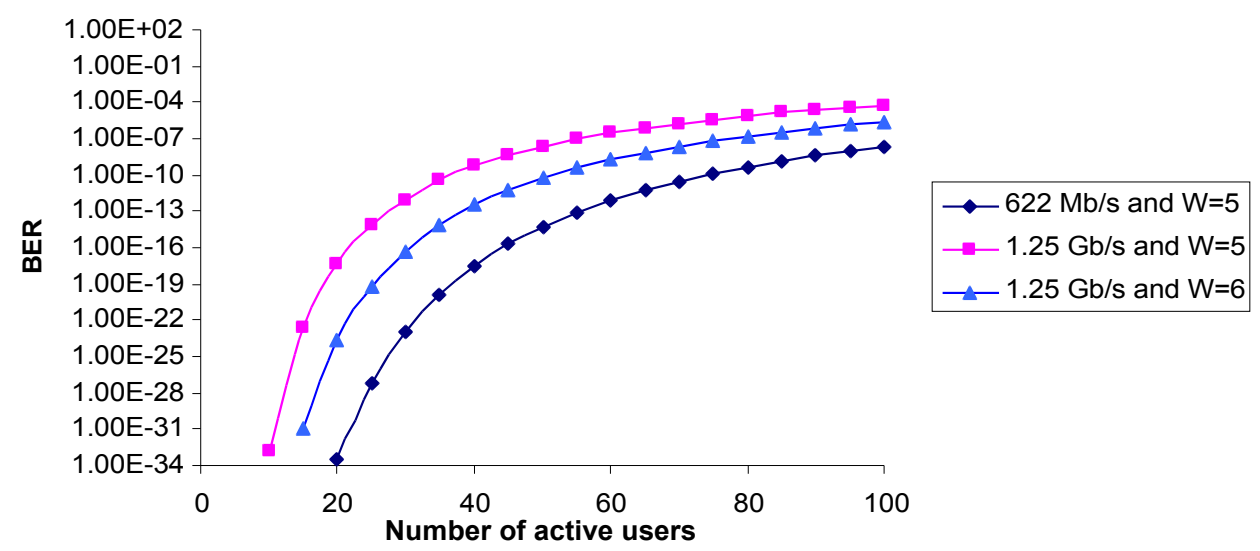

Fig. 7. BER versus the number of active users for different data rates for the hybrid DCS code system at $P_{s r}=0 \mathrm{dBm}$. 
It is obvious that the BER variations observed with increasing number of users and increasing data rates result from the effects of two- and third-tone inter-modulation distortions of the third order. On the other hand, the number of users with an acceptable BER (the maximum $10^{-9}$ ) is 35 for the minimum weight number and $1.25 \mathrm{~Gb} / \mathrm{s}$. It should be noted that, although the BER can go down when the weight number increases up to six for $1.25 \mathrm{~Gb} / \mathrm{s}$, the number of active users then increases to 55 .

It is evident from Fig. 8 that the thermal noise represents a main factor that limits the system performance. We also see that the effect of the shot noise is smaller when compared to the thermal noise, at least at the power source $P_{s r}=-10 \mathrm{dBm}$.

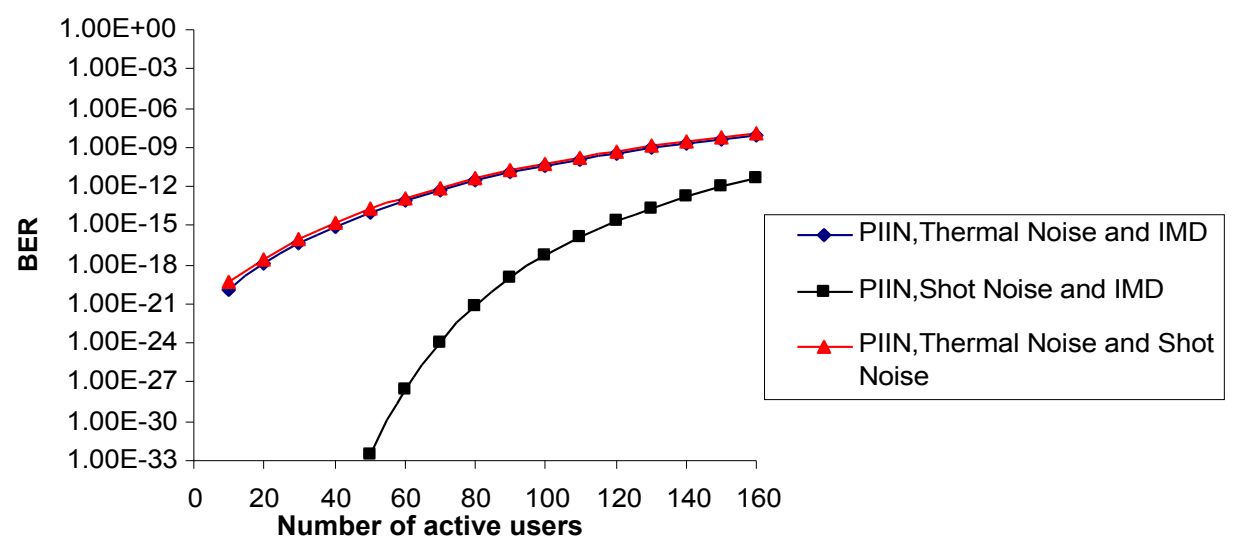

Fig. 8. Illustration of BER variations occurring with increasing number of active users for different noise conditions at $P_{s r}=-10 \mathrm{dBm}$.

\subsection{Simulation analysis}

The hybrid system has been simulated using the software "OptiSim ${ }^{T M}$ ", Version 9. The simulation is implemented for the two-subcarrier channel basing on the DCS code. Here we adopt the data rate $155 \mathrm{Mbit} / \mathrm{s}$ for each subcarrier channel. Furthermore, the subcarrier frequencies are set to be equal or larger than two times (the Niquest frequency) the bit rate. Each optical channel has the spectral width of $0.8 \mathrm{~nm}$. The simulation has been carried out for a standard single-mode optical fibre ITU-T G.652. All the parameters that describe the attenuation $(0.25 \mathrm{~dB} / \mathrm{km})$, the dispersion $(18 \mathrm{ps} /(\mathrm{nm} \times \mathrm{km})$, and the nonlinear effects (four-wave mixing and self-phase modulation), have been activated and specified according to their typical industrial values, in order to simulate a real environment as close as possible. The noise generated at the receivers has been set to be random and totally uncorrelated. The dark current value has been put to be $5 \mathrm{nA}$. We have also used the thermal noise coefficient $1.8 \times 10^{-23} \mathrm{~W} / \mathrm{Hz}$ for each of the photo-detectors. The performance of the system has been characterised by referring to the BER and the eye diagram pattern.

Fig. 9 shows the effect of fibre distance on the performance of our system having two subcarrier frequencies for different light source powers. It is clear that the dispersion has significant impact on the system performance when the fibre length increases. Our simulation results indicate that the system performance is deteriorating by about more than one order of magnitude, whenever the dispersion effect is activated in the simulation model. In addition, our results testify that the system performance is worsening as the fibre length increases from 20 to $50 \mathrm{~km}$.

Our system based on the DCS code achieves the BER $\leq 10^{-9}$ at $20,25,30$ and $35 \mathrm{~km}$ for the 
particular case of $622 \mathrm{Mb} / \mathrm{s}$, when the light power source is characterised by the value $-1 \mathrm{dBm}$. In fact, the light power source should increase with increasing fibre length, in order to recover degradation of the signal form. Thus, in order to design and optimise the link parameters, one should adopt as short a maximum fibre length as possible, for using low-power sources and achieving a desired system performance without any dispersion-compensating devices.

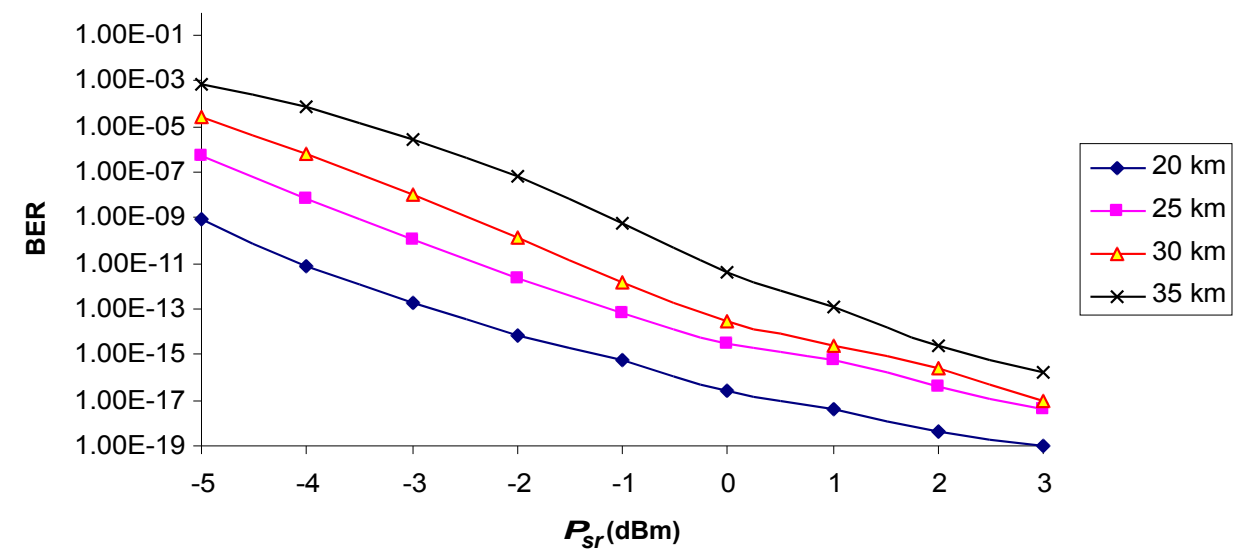

Fig. 9. Dependences of BER on the $P_{s r}$ parameter observed for different fibre lengths for the hybrid SCM/SAC-OCDMA.

\section{Conclusion}

In the present study we have analysed numerically the SCM/SAC-OCDMA system that uses the DCS code as a signature sequence code. The performance of the system is revealed to improve significantly, because the total loss is reduced as the AND detection technique requires less number of filters in the decoder. In addition, making use of the code words with less crosscorrelation value mitigates the PIIN, which improves the overall system performance.

The hybrid system based on the DCS code has also been simulated using the "OptiSim" software. The simulation results show that the system performance with the data rates of $622 \mathrm{Mb} / \mathrm{s}$ and $1.25 \mathrm{~Gb} / \mathrm{s}$ are affected by the attenuation the dispersion effect and the fibre length. We have found that $-1 \mathrm{dBm}$ is an optimum power of light source needed to achieve the minimum BER for the transmission fibre lengths of 20,25,30, and $35 \mathrm{~km}$. Consequently, use of the DCS code as a signature sequence for the case of SCM/SAC-OCDMA systems proves to be very promising solution. It suppresses the MAI, as compared to the system that uses the other SAC codes, and enables carrying large numbers of code words and subcarrier channels.

\section{References}

1. Khaleghi $\mathrm{F}$ and Kavehrad M, 1995. A subcarrier multiplexed CDM optical local area network, theory and experiment. IEEE Trans. Commun. 43: 75-87.

2. Yin H, Liang W, Ma L and Qin L, 2009. A new family of two-dimensional triple-code weight asymmetric optical orthogonal code for OCDMA networks. Chinese Opt. Lett. 7: 102-105.

3. Biao C, Fu-chang W, Jian-dong H and Sai-ling H, 2005. A novel OCDMA drop unit based on fiber gratings and 2D wavelength-time codes. Optoelectronics Lett. 1: 53-56.

4. Prucnal P R, Santoro M A and Fan T R, 1986. Spread spectrum fiber-optic local area network using optical processing. J. Lightwave Technol. 4: 547-554. 
5. Pfeiffer T, Deppisch B, Witte M and Heidemann R, 1999. Operational stability of a spectrally encoded optical CDMA system using inexpensive transmitters without spectral control. IEEE Photon. Technol. Lett. 11: 916-918.

6. Zouine Y, Dayoub I, Haxha S. B and Rouvaen J M, 2008. Analyses of constraints on high speed optical code division multiplexing access (OCDMA) link parameters due to fiber optic chromatic dispersion. Opt. Communs. 281: 1030-1036.

7. Stok A and Sargent E H, 2000. Lighting the local area: optical code-division multiple access and quality of service provisioning.. IEEE Network. 14: 42-46.

8. Noshad $\mathrm{M}$ and Jamshidi K, 2010. Code family for modified spectral-amplitude-coding OCDMA system and performance analysis. IEEE/OSA Journal of Opt. Commun. Netw. 2: 344-354.

9. Salehi J A, 1989. Code division multiple access technique in optical fiber networks- Part II: System performance analysis. IEEE Trans. Commun. 37: 834-842.

10. Yang G C and Kwong W C, Prime code with applications to CDMA optical and wireless networks. London: Artech House (2002).

11. Ahmad Anas S B, Abdullah M K, Mokhtar M, Aljunid S A and Walker S D, 2009. Optical domain service differentiation using spectral-amplitude-coding. Opt. Fiber Technol. 15: 2632.

12. Hasson F N, Aljunid S A, Samad M D A and Abdullah M K, 2008. Spectral amplitude coding OCDMA using AND subtraction technique. Appl. Opt. 47: 1263-1268.

13. Wei Z, Shalaby H M H and Shiraz H G, 2001. New code families for fiber-brag-gratingbased spectral-amplitude-coding optical CDMA systems. IEEE Photonics Technol. Lett. 13: 890-892.

14. Wei Z, Shalaby H M H and Shiraz H G, 2001. Modified quadratic congruence codes for fiber Bragg-grating based spectral-amplitude coding optical CDMA system. J. Lightwave Technol. 19: $1274-1281$.

15. Fadhil H A, Aljunid S A and Ahmed R B, 2009. Effect of random diagonal code link of an OCDMA scheme for high-speed access networks. Opt. Fiber Technol. 15: 237-241.

16. Aljunid S A, Ismail M and Ramil A R, 2004. A new family of optical code sequence for spectral-amplitude-coding optical CDMA systems. IEEE Photon. Technol. Lett. 16: 23832385.

17. Abd T H, Aljunid S A, Fadhil H A, Ahmed R B and Saad N M, 2011. Development of a new code family based on SAC-OCDMA system with large cardinality for OCDMA network. Opt. Fiber Technol. 17: 273-280.

18. Hui R, Zhu B, Huang R, Allen C T, Demarest K R and Richards D, 2002. Subcarrier multiplexing for high speed optical transmission. J. Lightwave Technol. 20: 417-427.

19. Sahbudin R K Z, Abdullah M K and Mokhtar M, 2009. Performance improvement of hybrid subcarrier multiplexing system using code for OCDMA systems using spectral direct decoding detection technique. Opt. Fiber Technol. 15: 266-273.

20. Koshy B J and Shankar P M, 1997. Efficient modeling and evaluation of fiber-fed microcellular networks in a land mobile channel using a GMSK modem scheme. IEEE J. Selected Areas in Communications. 15: 694-705.

21. Koshy B J and Shankar P M, 1999. Spread Spectrum techniques for fiber-fed microcellular networks, IEEE Transactions on Vehicular Technol. 48: 847-857. 


\begin{abstract}
Abd T. H., Aljunid S. A., Fadhil H. A., Ahmad R. B. and Junita M. N., 2012. Enhancement of performance of a hybrid SAC-OCDMA system using dynamic cyclic shift code. Ukr.J.Phys.Opt. 13: $12-27$.
\end{abstract}

Анотація. 3 використанням чисельних методів $і$ моделювання в роботі досліджено гібридну технологію мультиплексування піднесучої (SCM), включаючи спектральне кодування амплітуди і оптичний множинний доступ із кодовим розподілом каналів (SACOCDMA). Гібридні системи (SCM/SAC-OCDMA) поєднують дві вищезгадані схеми таким чином, щзо вони є стійкими до зовнішніх втручань і проявляють більшу швидкість передачі даних. Ми покращили ефективність роботи гібридної системи - SCM/SAC-OCDMA на основі спектрального кодування амплітуди для зменшення числа втручань $і$ поліпшення чутливості при коефіцієнті помилкових бітів $10^{-9}$. В результаті, запропонована нами система, яка базується на динамічному ичклічному коді, є ефективнішою у порівнянні зі стандартними системами (SAC-OCDMA) у яких використовується звичайний код. 